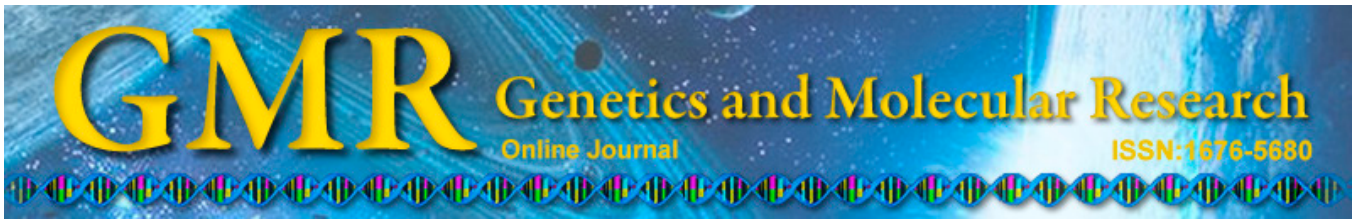

\title{
Screening of highly informative and representative microsatellite markers for genotyping of major cultivated cotton varieties
}

\author{
M. Kuang, W.H. Yang, F. Wang, H.X. Xu, Y.Q. Wang, D.Y. Zhou, \\ D. Fang, L. Ma and X.A. Feng \\ State Key Laboratory of Cotton Biology, Institute of Cotton Research, \\ Chinese Academy of Agricultural Sciences, Anyang, Henan, China \\ Corresponding author: W.H. Yang \\ E-mail: cottontest@126.com
}

Genet. Mol. Res. 13 (4): 9777-9786 (2014)

Received October 23, 2013

Accepted July 10, 2014

Published November 27, 2014

DOI http://dx.doi.org/10.4238/2014.November.27.5

\begin{abstract}
We screened and assessed published cotton simple sequence repeat (SSR) primers to establish a set of core SSR markers suitable for cotton major cultivars in China and analyzed genetic diversity based on the core marker set. Using a stepwise screening strategy, 12 leading cultivars for preliminary screening and 96 cultivars for rescreening were evaluated. A total of 184 polymorphic SSR markers were initially screened from 3299 candidates, and a core set of 52 SSR markers with wide genome coverage (2 markers per chromosome) was obtained. Among 96 major cultivars, 273 amplification genotypes were generated using the core marker set. Polymorphism information content values ranged from $0.28-0.83$, with an average value of 0.56 . The core SSR marker set detected on denaturing polyacrylamide gel electrophoresis indicated that the band genotype was either a single or double band on conventional cultivars, while most were double bands (65.4\%). Among 56 hybrids, the average heterozygosis rate was
\end{abstract}


$35.8 \%$, ranging from 7.1-55.4\%. Eighteen of 96 cultivars had distinct band genotypes. The genetic diversity analyzed using the of NTSYS-pc V2.10 software indicated that the Yangtze River valley cotton region had the highest polymorphic level, followed by Xinjiang and then the Yellow River valley. The genetic basis of conventional cultivars was narrower than that of hybrids. The core marker set can be used for fingerprint construction, variety identification, and purity tests of major cotton cultivars in China.

Key words: Genetic diversity; Core marker; Cotton; Major cultivar; Simple sequence repeat

\section{INTRODUCTION}

Cultivated cotton is the world's leading fiber crop and the second most important oilseed crop (Yu et al., 2012a). The authorization and release of high-quality cultivars is important in agricultural production. Recently, the use of various breeding parents has increased, resulting in genetic similarity among cotton cultivars and difficulty in distinguishing these cultivars based on phenotypic performance. Transgenic technology has added to the challenge because modifying a single trait can produce a new cultivar (Kuang et al., 2011). Traditional variety identification technology using the grow-out test involves growing plants to maturity and assessing several morphological characteristics that distinguish individual plants. Environmental influences on morphological characters and time factor make it difficult to collect morphological data; other limitations include unambiguous differentiation of genotypes (Ashok et al., 2011). These characteristics have created disorder in the cotton market and made the supervision of seed production and trade very difficult. Molecular markers can be used to distinguish between cultivars because they offer a fast and reliable technique for precisely assessing plant genotypes. Therefore, DNA fingerprint identification is important for ensuring that seed quality is robust.

Microsatellites, also known as simple sequence repeats (SSRs), have a number of advantages over many molecular markers, including their co-dominant inheritance, relative abundance, extensive genome coverage, ease detection by polymerase chain reaction (PCR), and reproducibility and locus specificity (Powell et al., 1996; Eillis and Burke, 2007). Over the last decade, thousands of SSRs have been identified (Blenda et al., 2006; Guo et al., 2007; Lacape et al., 2009; Yu et al., 2011, 2012a). These cotton SSR markers were developed either from random-enriched small genomic clones, large insert bacterial artificial chromosome clones, or expressed sequence tags (ESTs) of Gossypium species. Their use is limited by great variability among markers in polymorphisms, amplification quality, location, and distribution in the genome, and should be examined to determine their usefulness. Previous studies have demonstrated that the evaluation of primer polymorphism levels is greatly influenced by the material type and range of materials selected (Lübberstedt et al., 1998; Matsuoka et al., 2002). A core set of SSR markers was evaluated for DNA polymorphisms in 12 genotypes of 6 Gossypium species, collectively known as the cotton marker database panel. The results indicated that the core marker set was robust for characterization of Gossypium germplasm (Yu et al., 2012a). Although polymorphism levels for these primers 
are high in different Gossypium germplasms, levels are low in cultivated varieties such as upland cotton. However, most cotton major cultivars are upland cotton and have a narrow genetic base because of the factors described above. Thus, it is difficult to identify general SSRs for major cotton cultivars in China. This explains why variety identification technology based on SSR markers has not been widely applied in the cotton seed market. Selection of highly polymorphic primers in our study was based on common upland cotton varieties, making these primers significant for related studies and application to ordinary cultivars. In our study, 96 cultivars collected from 3 main cotton regions (Yangtze River valley, Yellow River valley, and Xinjiang cotton region), including 8 main cotton production provinces at the present stage were used for primer polymorphism evaluation. All samples were upland cultivars excepted for 1 Sea Island cotton cultivar from Xinjiang. We predicted that 2 markers per chromosome would be necessary to cover the whole genome. The objective of this study was to thoroughly screen and assess published cotton SSR primers to establish a set of core SSR primers suitable for major cotton cultivars in China. Genotype characteristics and genetic diversity were analyzed based on the set of core markers. This core marker set can be used in fingerprint construction, variety identification, and purity testing for major cotton cultivars in China.

\section{MATERIAL AND METHODS}

\section{Materials}

Ninety-six main cotton cultivars were used in this study, including 95 upland cotton varieties and 1 Gossypium barbadense variety coded 31 (Table 1). Twelve varieties from the Chinese National Crop Germplasm Repository (asterisk in Table 1) were used for preliminary screening. The 12 varieties were leading cultivars planted in 3 main cotton regions, including 4 cultivars from the Yangtze River valley, 5 cultivars from the Yellow River valley, and 3 cultivars from the Xinjiang cotton region.

All of the 96 cultivars were used for rescreening materials consisting of 40 conventional cultivars coded from 1-40 and 56 hybrid cultivars coded from 41-96. Ninety-six cultivars, except for 12 preliminary materials, were collected from 8 main cotton production provinces distributed in 3 main cotton regions in China.

\section{DNA extraction}

Cotton genomic DNA was extracted from dry seeds of each cultivar following a method described by Kuang et al. (2010). The quality and quantity of DNA were estimated by measuring optical density at $260 / 280 \mathrm{~nm}$ with an ultraviolet spectrophotometer.

\section{Screening of SSR primers}

SSR primer pairs used in this study were developed by different research groups in the cotton research community. The sequence of individual primer pairs and the source clone for each SSR can be found in the cotton marker database (http://www.cottonmarker.org/). All primers were synthesized by TaKaRa Company (Dalian, China). The 12 leading cultivars were used for preliminary screening and 96 cultivars were used for rescreening. 
Table 1. Ninety-six cotton cultivars used in this study.

\begin{tabular}{|c|c|c|c|c|c|c|c|}
\hline No. & Cultivar & No. & Cultivar & No. & Cultivar & No. & Cultivar \\
\hline $1 *$ & Lumianyan 21 & $25^{*}$ & Zhongmiansuo 49 & $49 *$ & Zhongmiansuo 63 & 73 & Ezamian 5 \\
\hline $2 *$ & Lumianyan 28 & $26^{*}$ & Xinluzao 33 & 50 & Jichuangmian 1 & 74 & Ezamian 6 \\
\hline $3^{*}$ & Zhongzhimian 2 & $27^{*}$ & Xinluzao 42 & 51 & Jimian 589 & 75 & Ezamian 14 \\
\hline $4^{*}$ & Zhongmiansuo 50 & 28 & Xinluzao 36 & 52 & Hengmian 4 & 76 & Ezamian 13 \\
\hline 5 & Zhongmiansuo 41 & 29 & Xinluzao 31 & 53 & Yuza 37 & 77 & Ezamian 16 \\
\hline 6 & Zhongmiansuo 45 & 30 & Xinluzhong 26 & 54 & Kaimian 5 & 78 & Jinzamian 288 \\
\hline 7 & Hanmian 103 & 31 & Xinhai 21 & 55 & Yuza 35 & 79 & Zhongkangza 1 \\
\hline 8 & Shikang 126 & 32 & Xinluzhong 33 & 56 & Lumianyan 30 & 80 & Xiangzamian 7 \\
\hline 9 & Guoxinmian 3 & 33 & Xinluzao 28 & 57 & Lumianyan 15 & 81 & Xiangnongmian 8 \\
\hline 10 & Chuangmian 3 & 34 & Xinluzhong 47 & 58 & Lumianyan 24 & 82 & Xiangzamian 3 \\
\hline 11 & Handan 284 & 35 & Xinluzao 48 & 59 & W8225 & 83 & Zhenghuamian 1 \\
\hline 12 & Jifeng 106 & 36 & Xinluzao 19 & 60 & Daiza 1 & 84 & Xiangzamian 8 \\
\hline 13 & Hanmian 103 & 37 & Xinluzao 12 & 61 & Wanmian 31 & 85 & Xiangzamian 16 \\
\hline 14 & Fengkangmian 1 & 38 & Xinluzhong 40 & 62 & Wanza 5 & 86 & Xiangfengmian 3 \\
\hline 15 & Jinke 178 & 39 & Guokangmian 1 & 63 & Quanyin 2 & 87 & Kemian 3 \\
\hline 16 & Jinmian 38 & 40 & Sikang 1 & 64 & Guofengmian 12 & 88 & Siza 3 \\
\hline 17 & Kuaifeng 868 & 41 & Zhongmiansuo 47 & 65 & Wanza 3 & 89 & Fumian 2 \\
\hline 18 & Yinshan 6 & 42 & Zhongmiansuo 48 & 66 & Wanza 8 & 90 & Nannong 6 \\
\hline 19 & Kaimian 21 & 43 & Zhongmiansuo 51 & 67 & Wanmian 25 & 91 & Nankang 9 \\
\hline 20 & Shannongshengmian 1 & 44 & Zhongmiansuo 59 & 68 & Wanmian 19 & 92 & Yankangza 1 \\
\hline 21 & Renhe 39 & $45^{*}$ & Zhongmiansuo 70 & 69 & Ezamian 26 & 93 & Xuza 3 \\
\hline 22 & Lumianyan 27 & $46^{*}$ & Ezamian 10 & 70 & Ezamian 17 & 94 & Suza 201 \\
\hline 23 & Xinqiu 1 & $47^{*}$ & Suza 3 & 71 & Ezamian 23 & 95 & Suzamian 66 \\
\hline 24 & Lumianyan 29 & $48 *$ & Xiangzamian 10 & 72 & Gangzamian 8 & 96 & Nannong 98-7 \\
\hline
\end{tabular}

*The 12 leading cotton cultivars used for preliminary screening.

\section{PCR and detection}

All PCR amplification was carried out in a total volume of $20 \mu \mathrm{L}$ containing $40 \mathrm{ng}$ genomic DNA, 1X PCR buffer, 1 U Taq DNA polymerase (TaKaRa), $1.5 \mathrm{mM} \mathrm{MgCl}, 0.15 \mu \mathrm{M}$ of each primer, and $0.1 \mathrm{mM}$ dNTPs. Amplification was performed under the following conditions: 4 min at $94^{\circ} \mathrm{C}$ for strand separation followed by 32 cycles of $45 \mathrm{~s} \mathrm{at} 94^{\circ} \mathrm{C}$ for denaturation, $45 \mathrm{~s}$ at $55^{\circ} \mathrm{C}$ for annealing, and $45 \mathrm{~s}$ at $72^{\circ} \mathrm{C}$ for primer extension, and a final extension at $72^{\circ} \mathrm{C}$ for 7 min. Amplified PCR products were separated on $6 \%$ denaturing PAGE at $90 \mathrm{~W}$ for $1 \mathrm{~h}$. Silver staining method followed the method described by Wang et al. (2004a).

\section{Statistical analysis}

The genetic diversity of each SSR locus was obtained by calculating the frequency of the genotype based on polymorphism information content (PIC) following the formula developed by Anderson et al. (1993). A similarity matrix was constructed using Nei's genetic distance, and Nei's genetic distance were determined using NTSYSpc ver. 2.10 (NTSYS-PC 2.10, Applied Biostatistics, Setauket, NY, USA). The dendrogram showing relatedness among the 96 cotton cultivars based on Nei's genetic distance was constructed using unweighted pair group method with arithmetic means.

\section{RESULTS}

\section{SSR polymorphism}

A total of 184 polymorphic SSR markers were initially screened out of 3299 candidates assessed by 12 leading cultivars. Next, 184 polymorphic SSR markers were distributed 
on 26 chromosomes of the cotton genome, with an average of 7 SSR markers on each chromosome, ranging from 3-13. The difference may be related to different recombination rates of different chromosomal regions. Chromosome 5(c05) showed the most abundant polymorphic SSR markers with 13. Based on the genetic map by Yu et al. (2012b), c05 showed the longest genetic distance. Among the 12 leading cultivars, a total of 559 polymorphic genotypes were detected by 184 SSR markers, with an average of 3.04 genotypes per SSR marker, ranging from 2-6.

Two SSR markers were identified from each of the 26 cotton chromosomes to ensure even distribution of the markers on the cotton genome, giving a total of 52 core SSR markers (Table 2). Those with high polymorphisms and good repeatability were successfully rescreened out from 184 SSR markers assessed by 96 main cotton cultivars, including 12 leading cultivars. The set of core SSR markers consisted of 27 pair EST-SSRs and 25 pair genomic-SSRs. Among the 96 main cultivars, the number of amplification genotypes generated using the set of core SSR markers was 273, including 130 genotypes generated by EST-SSR and 143 genotypes generated by genomic-SSR. The results suggest that each EST-SSR can detect an average of 4.81 genotypes and each genomic-SSR can detect 5.72 genotypes.

Table 2. List of core SSR markers and information.

\begin{tabular}{|c|c|c|c|c|c|c|c|c|c|c|c|}
\hline Primer & Type & $\begin{array}{c}\text { Map } \\
\text { location }\end{array}$ & $\begin{array}{c}\text { No. of } \\
\text { genotypes }\end{array}$ & PIC & $\begin{array}{l}\text { Heterozygosis } \\
\text { rate }(\%)\end{array}$ & Primer & Type & $\begin{array}{c}\text { Map } \\
\text { location }\end{array}$ & $\begin{array}{c}\text { No. of } \\
\text { genotypes }\end{array}$ & PIC & $\begin{array}{l}\text { Heterozygosis } \\
\text { rate }(\%)\end{array}$ \\
\hline NAU3254 & EST & 1 & 4 & 0.50 & 35.7 & CIR246 & Genomic & 14 & 7 & 0.71 & 42.9 \\
\hline BNL3085 & Genomic & 1 & 4 & 0.59 & 42.9 & NAU1070 & EST & 14 & 4 & 0.54 & 35.7 \\
\hline NAU3419 & EST & 2 & 5 & 0.51 & 23.2 & BNL3033 & Genomic & 15 & 4 & 0.54 & 32.1 \\
\hline NAU2277 & EST & 2 & 4 & 0.45 & 41.1 & HAU1001 & EST & 15 & 5 & 0.51 & 36.4 \\
\hline NAU3995 & EST & 3 & 9 & 0.78 & 44.6 & BNL1026 & Genomic & 16 & 4 & 0.43 & 21.4 \\
\hline NAU1071 & EST & 3 & 4 & 0.57 & 35.7 & JESPR292 & Genomic & 16 & 3 & 0.51 & 35.7 \\
\hline HAU1300 & EST & 4 & 4 & 0.40 & 14.3 & HAU2786 & EST & 17 & 9 & 0.78 & 44.6 \\
\hline MUCS101 & EST & 4 & 3 & 0.56 & 45.1 & HAU2014 & EST & 17 & 3 & 0.59 & 44.6 \\
\hline NAU6094 & EST & 5 & 4 & 0.57 & 36.7 & TMB1638 & Genomic & 18 & 15 & 0.83 & 26.7 \\
\hline NAU1200 & EST & 5 & 4 & 0.50 & 41.1 & TMB2295 & Genomic & 18 & 5 & 0.46 & 27.3 \\
\hline DPL0238 & Genomic & 6 & 4 & 0.56 & 35.7 & NAU1102 & EST & 19 & 4 & 0.54 & 51.8 \\
\hline DPL0811 & Genomic & 6 & 5 & 0.56 & 39.3 & DPL0556 & Genomic & 19 & 5 & 0.52 & 21.4 \\
\hline NAU1362 & EST & 7 & 8 & 0.67 & 53.6 & BNL3948 & Genomic & 20 & 4 & 0.55 & 30.4 \\
\hline NAU1085 & EST & 7 & 3 & 0.57 & 44.6 & GH277 & Genomic & 20 & 6 & 0.66 & 42.6 \\
\hline DPL0133 & Genomic & 8 & 7 & 0.51 & 17.9 & DPL0376 & Genomic & 21 & 4 & 0.54 & 37.5 \\
\hline NAU1369 & EST & 8 & 3 & 0.34 & 16.7 & NAU1103 & EST & 21 & 4 & 0.58 & 34.5 \\
\hline NAU859 & EST & 9 & 7 & 0.54 & 39.3 & CGR6410 & Genomic & 22 & 4 & 0.58 & 35.7 \\
\hline CGR5707 & Genomic & 9 & 4 & 0.29 & 17.9 & NAU5099 & EST & 22 & 7 & 0.49 & 7.1 \\
\hline NAU1233 & EST & 10 & 7 & 0.70 & 43.9 & BNL3173 & Genomic & 23 & 4 & 0.28 & 12.5 \\
\hline DPL0296 & Genomic & 10 & 4 & 0.49 & 33.9 & BNL3140 & Genomic & 23 & 5 & 0.57 & 40.0 \\
\hline DPL0528 & Genomic & 11 & 10 & 0.71 & 50.0 & NAU0478 & EST & 24 & 5 & 0.57 & 33.9 \\
\hline HAU2026 & EST & 11 & 4 & 0.59 & 42.9 & NAU1125 & EST & 24 & 5 & 0.58 & 44.6 \\
\hline DPL0917 & Genomic & 12 & 10 & 0.79 & 44.6 & CGR6932 & Genomic & 25 & 7 & 0.57 & 28.6 \\
\hline BNL3261 & Genomic & 12 & 5 & 0.60 & 43.4 & HAU2022 & EST & 25 & 3 & 0.50 & 37.5 \\
\hline CER0168 & EST & 13 & 4 & 0.60 & 55.4 & CIR170 & Genomic & 26 & 3 & 0.52 & 33.9 \\
\hline BNL1421 & Genomic & 13 & 9 & 0.72 & 48.2 & MGHES44 & EST & 26 & 4 & 0.39 & 33.9 \\
\hline
\end{tabular}

Table 2 shows the PIC values of each core SSR marker among the 96 main cotton cultivars. The level of genetic diversity between Gossypium hirsutum and G. barbadense detected using the core SSR marker set was higher than that within the G. hirsutum genotypes. PIC values of the core SSR markers set on the 96 main cotton cultivars ranged from 0.28-0.83, with an average value of 0.56 . Most SSRs from the core SSR marker set were highly informative $(\mathrm{PIC}>0.5)$ and 8 SSR markers were moderately informative $(0.3<\mathrm{PIC}<0.5)$, while only 2 were slightly informative (PIC $<0.3$ ). 


\section{Genotype characteristics}

The core SSR marker set detected on denaturing PAGE showed either a single band or double band for 40 conventional cultivars, with most appearing as double bands $(65.4 \%)$. The results are consistent with the genetic characterization of cotton showing that the A-genome and D-genome were of high homology. Figure 1 shows the DNA fragments amplified by the core SSR marker NAU1102 in 96 major cotton cultivars.

Combined with the band type displayed for the 56 hybrid and 40 conventional cultivars, 3 heterozygous band types of hybrids showed the following patterns: 1) double band in the hybrid, with a single band in conventional cultivars; 2) triple band in the hybrid, with a double band in conventional cultivars; 3) 4 bands in the hybrid, a double band in conventional cultivars. The second type accounted for $57.7 \%$, followed by the first type, accounting for $34.6 \%$, while the third type accounted for only $9.61 \%$. Among 56 hybrid cultivars, the average heterozygosity rate of the core marker set was $35.8 \%$, ranging from $7.1-55.4 \%$ (Table 2). The marker referred to as CER0168 showed the highest heterozygosity rate; this marker can be considered to be the preferred primer in the hybrid seed purity test.

Eighteen of 96 cultivars showed distinct band genotypes amplified by 17 specific primers. The unique sea island cotton cultivar known as Xinhai2 1 had the most abundant specific primers, with 32 of 52 core markers, accounting for $61.5 \%$. The result is consistent with the significantly different genetic backgrounds between upland cotton and sea island cotton. Among other 17 upland cotton cultivars, 2 cultivars had 3 specific primers, 3 cultivars had 2 specific primers, and 12 cultivars had 1 specific primer (Table 3 ). These specific primers can be directly applied for variety identification for the corresponding cultivars.

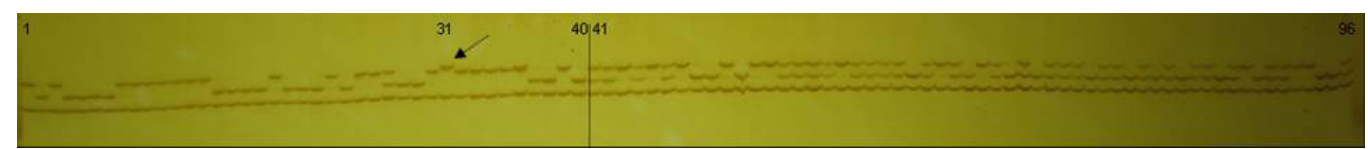

Figure 1. DNA fragments amplified by core SSR marker NAU1102 in 96 major cotton cultivars. Lanes 1-40= conventional cultivar; lanes 41-96 = hybrid cultivar; lane $31=$ sea island cotton.

Table 3. Cotton cultivars with specific primers.

\begin{tabular}{llll}
\hline Cultivar & Specific primer & Cultivar & Specific primer \\
\hline Zhongmiansuo 59 & NAU859, BNL1421,TMB1638 & Suza 201 & NAU3995, HAU2786, CGR6932 \\
Xinluzao 36 & NAU859, HAU1001 & Siza 3 & NAU1102, TMB1638 \\
Yankangza 1 & DPL0528, BNL3140 & Zhongmiansuo 41 & DPL0133 \\
Guoxinmian 3 & DPL0811 & Yinshan 6 & BNL3261 \\
Shannongshengmian 1 & TMB2295 & Xinluzao 33 & BNL1421 \\
Zhongmiansuo 51 & TMB1638 & W8225 & DPL0917 \\
Wanzamian 31 & NAU1362 & Wanmian 19 & NAU859 \\
Ezamian 14 & NAU1125 & Xiangnongmian 8 & CGR6932 \\
Xiangzamian 8 & DPL0528 & & \\
\hline
\end{tabular}

\section{Genetic distance and diversity}

SSR amplification results were used to generate a genetic similarity matrix. Genetic similarity coefficients among 96 main cultivars ranged from 0.6300 to 0.9927 , with an average of 0.7660 , indicating that the cultivars we used had a vast genetic base. The average genetic 
similarity coefficient of 12 leading cotton varieties used for preliminary screening was 0.7769 , suggesting that 12 leading cultivars were well-represented. The genetic diversity analyzed using the NTSYS-pc V2.10 software indicated that Yangtze River valley cotton region had the highest polymorphic level, followed by Xinjiang cotton region, and then Yellow River valley (Table 4). Forty-six cultivars, except for 2 from the Yangtze River valley, were hybrids, while all cultivars from Xinjiang were conventional cultivars. There were 36 cultivars from the Yellow River valley, including 24 conventional cultivars, accounting for two-thirds, and 12 hybrids accounting for one-third. The average genetic similarity coefficients of 40 conventional cultivars and 56 hybrids were 0.8122 and 0.7617 , respectively. The results indicated that the genetic basis of conventional cultivars was narrower than that of hybrids.

The general cluster of 96 cultivars was analyzed using the unweighted pair-group method with arithmetic means based on similarity coefficients (Figure 2). The unique sea island cotton coded 31 was clustered in an individual group. Another 95 upland cotton cultivars were clustered in 2 groups. The first group consisted of 56 cultivars, including 37 conventional cultivars and 19 hybrids, while 12 hybrids were clustered together. The second group consisted of 39 cultivars and the entire group except for 2 cultivars included hybrids, indicating that the same types of cultivars were clustered together in some cases.

\section{Table 4. Genetic diversity of 3 main cotton regions.}

\begin{tabular}{llccc}
\hline Cotton region & Maximum & Minimum & No. of cultivars & Average of similarity coefficient \\
\hline Yangtze River valley & 0.9450549 & 0.6739927 & 46 & 0.7618 \\
Yellow River valley & 0.992674 & 0.6776557 & 36 & 0.8071 \\
Xinjiang & 0.9267399 & 0.6446886 & 14 & 0.7892 \\
\hline
\end{tabular}

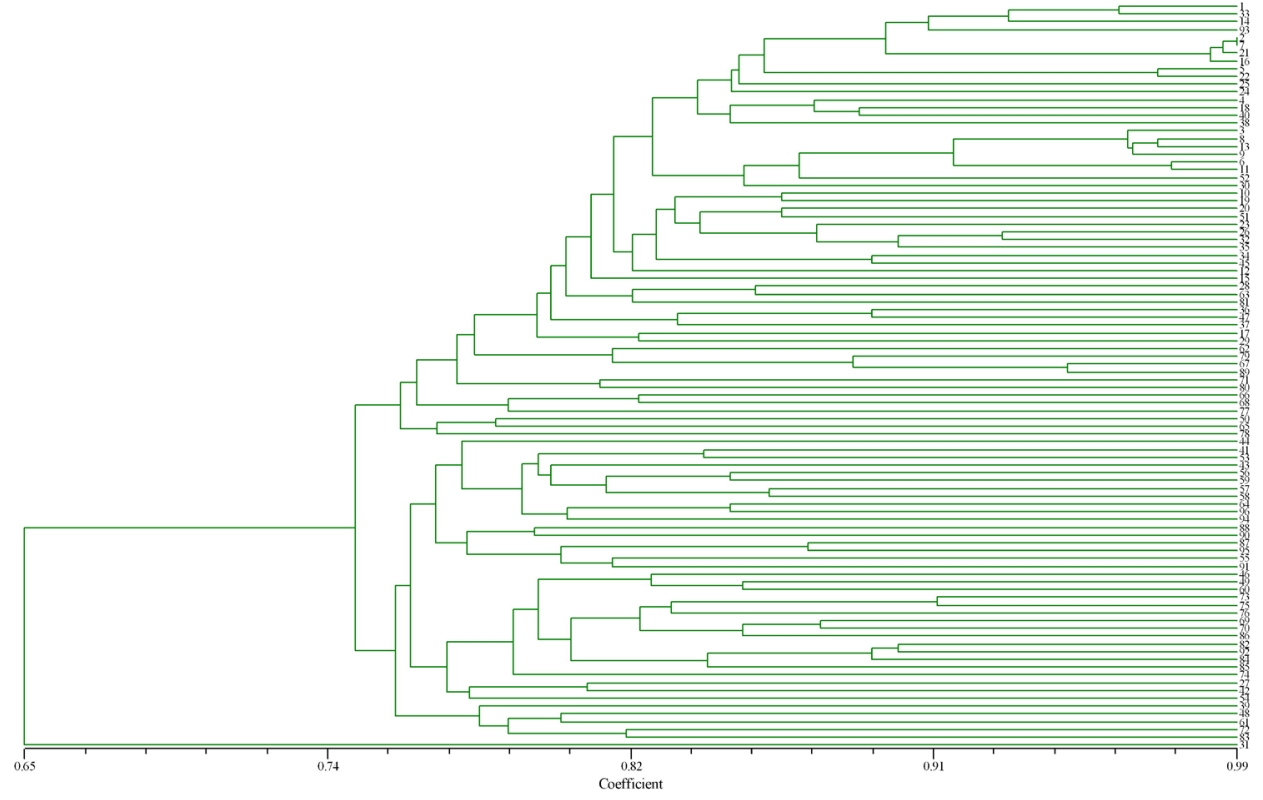

Figure 2. Unweighted pair group method with arithmetic mean clustering analysis of 96 major cotton cultivars. 
Figure 2 shows that 3 cultivars, including Lumianyan 28, Hanmian 802, and Renhe 39, were very closely related, with similarity coefficients of $0.992674,0.992674$, and 0.985348 , respectively. Genetic genealogy indicated that both Lumianyan 28 and Hanmian 802 were related to Zhongmiansuo 12, sharing some morphological traits such as growth period, plant type, leaf type, and boll type. Both Hanmian 802 and Renhe 39 were the offspring of Simian 3.

\section{DISCUSSION}

The development and selection of a set of core SSR primers are important for variety purity determination, identity detection, and DNA fingerprint database construction to ensure robust quality seed in cotton cultivars. In previous studies, accessions with significantly different phenotypes and genetic backgrounds were used for primer screening, and the obtained markers were applied for genetic diversity assessment, genetic map construction, quantitative trait loci mapping, etc. (Bertini et al., 2006; Chen and Du, 2006; Pang et al., 2006; Guo et al., 2007; Pan et al., 2008; Yu et al., 2012b). These markers may represent polymorphisms between different Gossypium species. However, the ability to distinguish between upland cottons is poor, particularly among upland cotton cultivars. To obtain a set of SSR core primers of cotton cultivars, a stepwise screening strategy was adopted. First, 12 leading cotton cultivars from 3 main cotton regions were used for preliminary screening. Subsequently, 96 main cultivars collected from 8 main cotton production provinces were used for rescreening. In this study, we proposed and reported a core set of 52 SSR markers with wide genome coverage of 2 evenly distributed markers per chromosome for 26 chromosomes. The results indicated that most markers were highly informative for the 96 main cotton cultivars. The core SSR marker set was suitable for identifying main cotton cultivars at the current stage in China and will play an important role in fingerprint construction, seed genuineness, and purity identification of the main cotton cultivars.

Previous studies have demonstrated that the polymorphism level of SSR primers is associated with the number and the type of repeated units, genomic region, database source for primer development, and materials used for detection (Wang et al., 2007). Dong (2007) studied the genetic diversity of 4 cultivated species using SSR markers and demonstrated that the SSR loci distributed on different chromosomes were at different evolutionary levels. Macaulay et al. (2001) studied SSR primers in barley and found uneven SSR distribution in the genome. Our results also suggest that the distribution of polymorphism SSR primers was uneven in the cotton genome. Uneven distribution of SSR primers throughout the genome is an obstacle for selecting a set of core markers. Thus, we reduced the stringency of selection with low primer density to ensure an even distribution of primers on chromosomes.

Yang et al. (2005) used genomic-SSR and EST-SSR markers to measure the genetic diversity among the hexaploid wheat population; the results showed that the polymorphism revealed by EST-SSR was lower than that by genomic-SSR. Studies of other crops also demonstrated that polymorphism levels were generally lower in primers developed from ESTs with unknown functions and genes with known functions compared to those developed from the entire genome (Leigh et al., 2003; Ghislain et al., 2004). Our results suggest that polymorphisms of genomic-SSR were higher than those of EST-SSR in 96 main cotton cultivars. The polymorphism level of SSR primers was generally higher in non-coding regions than in coding regions. This may be the results of a decrease in allele combinations, resulting in decreases in the polymorphism level of linked SSR markers. 
There are 3 methods available for DNA fingerprinting, including the distinct band method, primer combination method, and core primer combination method (Wang et al., 2003). In our study, 18 of 96 cultivars had distinct band genotypes amplified by 17 specific primers. The 18 cultivars could be identified using only 1 corresponding specific primer. However, most (78 of 96) cultivars showed no distinct band genotypes and the distinct band method was useful only in fixed materials. When the materials were enlarged, samples originally showing a distinct band genotype no longer showed a distinct band genotype. The primer combination method could discriminate all materials by different primers' limited combinations, avoiding a large amount of primer-screening. In our study, a core set of 52 SSR markers with wide genome coverage were successfully screened. Data from different laboratories can be compared and integrated using a set of fixed core primer combination, standardizing cotton DNA fingerprint analysis methods.

The genetic diversity analyzed using the NTSYS-pc V2.10 software indicated that Yangtze River valley cotton region had the highest polymorphic level, followed by Xinjiang, with the lowest from the Yellow River valley. The genetic basis of conventional cultivars was narrower than that of hybrids. However, Liu et al. (2003) suggested that the genetic basis of hybrids were narrower than that of conventional cultivars. Both Xu et al. (2001) and Wang et al. (2004b) suggested that the genetic basis of the Yellow River valley cultivars was similar to that of Yangtze River valley. These results of these studies differed from our results. The reason for this difference may be that the genetic basis of hybrids was improved because of the development of hybrids through broad selection of parent and intermingles of unknown consanguinity during breeding process in recent years. The genetic relationships of cultivars derived from the same cotton region were similar to a certain extent. These results reflected the differences among different ecological regions with different breeding goals. Specifically, most cultivars from the Yangtze River valley and most cultivars from the Yellow River valley were clustered together, while cultivars from Xinjiang cotton region were spread throughout the Yellow River valley. These results are consistent with the results of Bie et al. (2001) and Bai et al. (2012).

\section{ACKNOWLEDGMENTS}

Research supported by the Ministry of Agriculture, China, the Basic R\&D Operation Special Fund for the Central Level, Non-Profit, Scientific Research Institutes, China (\#SJA1204), and the National Science and Technology Support Program, China (\#2011BAD35B09). We thank the cooperative members of the cotton research community, Shandong Cotton Center, Nanjing Agricultural University, and Huazhong Agricultural University for providing some of the SSR primers.

\section{REFERENCES}

Anderson JA, Churchill GA, Autrique JE, Tanksley SD, et al. (1993). Optimizing parental selection for genetic linkage maps. Genome 36: 181-186.

Ashok BD, Mamta PR, Manoj RB and Kshanada JM (2011). Identification and genetic purity testing of cotton F1 hybrid using molecular markers. India J. Biotechnol. 10: 301-306.

Bai J, Nie YC, Lin ZX, Guo XP, et al. (2012). Screening and evaluation of SSR core primers for identification of cotton hybrids. Cotton Sci. 24: 207-214.

Bertini C, Schuster I, Barros E, Moreira M, et al. (2006). Characterization and genetic diversity analysis of cotton cultivars 
using microsatellites. Genet. Mol. Res. 29: 321-329.

Bie S, Kong FL, Zhou YY, Zhang GM, et al. (2001). Genetic diversity analysis of representative elite cotton varieties in three main cotton regions in China by RAPD and its relation with agronomic characteristics. Sci. Agric. Sin. 34: 597-603.

Blenda A, Scheffler J, Scheffler B, Palmer M, et al. (2006). CMD: a cotton microsatellite database resource for Gossypium genomics. BMC Genomics 7: 132.

Chen G and Du XM (2006). Genetic diversity of source germplasm of Upland cotton in China as determined by SSR marker analysis. Yi. Chuan Xue Bao. 33: 733-745.

Dong W (2007). The Analysis of Genetic Diversity and SSR Abundance in Cotton Germplasms. Master's thesis, Chinese Academy of Agriculture Sciences, Beijing.

Eillis JR and Burke JM (2007). EST-SSRs as a resource for population genetic analyses. Heredity 99: 125-132.

Ghislain M, Spooner DM, Rodriguez F, Villamon F, et al. (2004). Selection of highly informative and user-friendly microsatellites (SSRs) for genotyping of cultivated potato. Theor. Appl. Genet. 108: 881-890.

Guo WZ, Cai CP, Wang CB, Han ZG, et al. (2007). A microsatellite-based, gene-rich linkage map reveals genome structure, function and evolution in Gossypium. Genetics 176: 527-541.

Kuang M, Yang WH, Xu HX, Wang YQ, et al. (2010). A rapid method of DNA extraction from single cotton seed. Mol. Plant Breed. 8: 827-831.

Kuang M, Yang WH, Xu HX, Wang YQ, et al. (2011). Construction of DNA fingerprinting and analysis of genetic diversity with SSR markers for cotton major cultivars in China. Sci. Agric. Sin. 44: 20-27.

Lacape JM, Jacobs J, Arioli T, Derijcker R, et al. (2009). A new interspecific, Gossypium hirsutum x G. barbadense, RIL population: towards a unified consensus linkage map of tetraploid cotton. Theor. Appl. Genet. 119: 281-292.

Leigh F, Lea V, Law J, Wolters P, et al. (2003). Assessment of EST- and genomic microsatellite markers for variety discrimination and genetic diversity studies in wheat. Euphytica 133: 359-366.

Liu WX, Kong FL, Guo ZL, Zhang QY, et al. (2003). An analysis about genetic basis of cotton cultivars in China since 1949 with molecular markers. Yi. Chuan Xue Bao. 30: 560-570.

Lübberstedt T, Dussle C and Melchinger AE (1998). Application of microsatellites from maize to teosinte and other relatives of maize. Plant Breed. 117: 447-450.

Macaulay M, Ramsay L, Powell W and Waugh R (2001). A representative, highly informative'genotyping set'of barley SSRs. Theor. Appl. Genet. 102: 801-809.

Matsuoka Y, Mitchell SE, Kresovich S, Goodman M, et al. (2002). Microsatellites in Zea - variability, patterns of mutations, and use for evolutionary studies. Theor. Appl. Genet. 104: 436-450.

Pan ZE, Sun JL, Wang XW, Jia YH, et al. (2008). Screening of SSR core primers with polymorphism on a cotton panel. Biodivers. Sci. 16: 555-561.

Pang CY, Du XM and Ma ZY (2006). Evaluation of the introgressed lines and screening for elite germplasm in Gossypium. Chin. Sci. Bull. 51: 304-312.

Powell W, Machray GC and Provan J (1996). Polymorphism revealed by simple sequence repeats. Trends Plant Sci. 1: 215-222.

Wang FG, Zhao JR, Guo JL, She HD, et al. (2003). Comparison of three DNA fingerprint analyzing methods for maize cultivars' identification. Mol. Plant Breed. 1: 655-661.

Wang FG, Zhao JR, Guo JL, She HD, et al. (2004a). An improved PAGE/rapid silver staining method used in maize SSR markers. J. Agric. Biotechnol. 12: 606-607.

Wang FG, Zhao JR, Dai JR, Yi HM, et al. (2007). Selection and development of representative simple sequence repeat primers and multiplex SSR sets for high throughput automated genotyping in maize. Chin. Sci. Bull. 52: 215-223.

Wang XF, Zhang GY, Li XH, Li RQ, et al. (2004b). AFLP analysis of cotton with fusarium and verticillium wilts from the Huanghe and Changjiang valleys. Yi. Chuan Xue Bao. 31: 1426-1433.

$\mathrm{Xu}$ QH, Zhang XL and Nie YC (2001). Genetic diversity evaluation of cultivars (G. hirsutum L.) from the Changjiang River valley and Yellow River valley by RAPD markers. Acta Genet. Sin. 28: 683-690.

Yang XQ, Liu P, Han ZF, Ni ZF, et al. (2005). Genetic diversity revealed by genomic-SSR and EST-SSR markers among common wheat, spelt and compactum. Prog. Nat. Sci. 15: 24-33.

Yu JZ, Fang DD, Kohel RJ, Ulloa M, et al. (2012a). Development of a core set of SSR markers for the characterization of Gossypium germplasm. Euphytica 187: 203-213.

Yu JZ, Kohel RJ, Fang DD, Cho J, et al. (2012b). A high-density simple sequence repeat and single nucleotide polymorphism genetic map of the tetraploid cotton genome. G3 2: 43-58.

Yu Y, Yuan DJ, Liang SG, Li XM, et al. (2011). Genome structure of cotton revealed by a genome-wide SSR genetic map constructed from a BC1 population between Gossypium hirsutum and G. barbadense. BMC Genomics 12: 15.

Genetics and Molecular Research 13 (4): 9777-9786 (2014)

CFUNPEC-RP www.funpecrp.com.br 